\title{
Bioéthique et recherches thérapeutiques : éclairage d'un consentement
}

L'essai clinique contrôlé classique avec tirage au sort des traitements administrés est confronté aujourd'hui à une évolution rapide des mentalités, hâtée par l'épidémie de SIDA. Dans tous les cas où est testé un nouveau produit censé s'opposer à une affection grave ne bénéficiant pas de traitement efficace, le consentement libre et éclairé des malades requis pour la conduite de l'essai n'aboutira-t-il pas au désir de ces malades de choisir leur traitement, c'est-à-dire de disposer du produit à l'essai ? Il reviendra alors aux médecins et aux statisticiens de s'adapter à cette nouvelle demande. En France, la loi Huriet définit, pour la première fois dans ce pays, un cadre légal pour les essais thérapeutiques, établissant le concept d'équipes d'expérimentateurs agréés, travaillant sous le contrôle de " comités de protection des personnes ". Les évolutions en cours et la pression des partenaires économiques influent sans nul doute sur les conditions de l'application de cette loi.

\section{Claude Jasmin}

\section{ADRESSE}

C. Jasmin: chef du service d'hématologie, d'immunologie et de biologie des tumeurs, directeur de l'unité d'oncogenèse appliquée, de l'Inserm. Inserm U. 268, groupe hospitalier PaulBrousse, 14, avenue Paul-VaillantCouturier, 94800 Villejuif, France. orsque Joseph Meister fut conduit devant Louis Pasteur après avoir été mordu par un chien présumé enragé, le savant hésita à le traiter. Il consulta deux de ses collègues médecins et ne prit sa décision qu'après avoir acquis la certitude que la mort de l'enfant était inévitable en l'absence de traitement. L'essai thérapeutique apparaît comme une obligation pour le médecin appelé à se confronter à l'échec des traitements connus. Le "mieux-faire " est son obsession.

L'évolution de la pratique de l'essai thérapeutique sous l'impulsion des biostatisticiens vers l'incontournable essai contrôlé, souvent "randomisé "*, a compliqué la relation médecin-malade. Le patient reçoit le traitement par hasard mais non par choix, forçant ainsi le médecin à lui avouer son ignorance même relative. S'agit-il plus des progrès de la médecine que de résoudre au mieux son propre cas?

* Essai randomisé : randomisé, de random (hasard), est un anglicisme qui s'applique aux essais contrôlés en simple ou double insu, avec tirage au sort du type de traitement administré. 


\section{RÉFÉRENCES}

1. Appelbaum S, Grisso $\mathrm{T}$. Assessing patients' capacities to consent to treatment. $N$ Engl J Med 1988 ; 319 : 1635-8.

2. Moore MJ, O'Sullivan B, Tannock IF How expert physicians would wish to be treated if they had genitourinary cancer. $J$ Clin Oncol 1988 ; 6 : 1736-45

3. Rigo G. Le consentement, support du contrat entre le malade et le médecin. Med Hyg 1986 ; 44 : 2008-13.

4. Sider RC, Clements CD. The new medical ethics. Arch Intern Med $1985 ; 145$ : 2169-71.

5. Zelen M. A new design in randomized clinical trials. $N$ Engl J Med $1979 ; 300$ : 1242-5.

6. Kass L. Ethical dilemmas in the care of the ill : 1 . What is the physician service? JAMA 1980 ; 244: 1811-6.

7. Editorial. Consent how informed ? Lancet $1984 ; 1: 1445-7$.

8. Lantos J, Siegler M, Cutler L. Ethical issues in growth hormone therapy. JAMA $1989 ; 261$ : 1020-4.

9. Brett AS. Treating hypercholesterolemia. How should practicing physicians interpret the published data for patients. $N$ Engl J Med 1989 ; 321 : 676-80.

10. Edgar H, Rothman J. Rights of access and protection. New rules of new drugs : the challenge of AIDS to the regulatory process. Milbank Quart 1990;68: 111-42.

11. Shapiro MF, Charrow RP. Special report. Scientific misconduct in investigational drug trials. $N$ Engl J Med 1985 ; 312 : 731-6.

12. Beecher H. Ethics and human experimentation. $N$ Engl J Med 1987 ; 317 . 1195-9.

13. Rothman DJ. Ethics and human experimentation. Henry Beecher revisted. $N$ Engl J Med 1987; 317 : 1195-8.

14. Fontenay M, Mettetal JF. Protocole ouvert sur la ddI aux États-Unis. 290 décès et des effets toxiques qui incitent à la prudence vis-à-vis de la ddI. SIDA $1990 ; 90$ : 35-6.

15. Lind SE. Finder's fees for research sub474
Qui consent? A quoi ? Pour qui ? Pour quoi ? Ces questions toujours présentes, parfois formulées, se posent au médecin aussi bien dans sa pratique courante que dans le cadre d'une recherche thérapeutique.

L'espace exploré fait partie de notre environnement socioculturel dans tout pays soucieux de la protection du citoyen, qu'il soit anglo-saxon, accordant la priorité au droit de chaque individu de disposer de son corps (Habeas Corpus), ou privilégiant une vision plus collective des droits de l'homme. Sur notre scène se trouvent le malade, à la recherche du mieuxêtre, et le médecin, soucieux du bienfaire, acteurs principaux d'enjeux juridiques, sociaux, économiques et bien entendu médico-scientifiques.

L'un éclaire, l'autre consent. Comment le médecin choisit-il l'éclairage, fort ou faible, large ou limité. Comment règle-t-on ce geste important ? Que représente-t-il pour le praticien dans sa relation avec le patient ? Ce dernier consent-il librement? Avec ou sans mot dire?

L'éclairage d'un consentement, aussi intense soit-il, préservera toujours des zones d'ombre que nous n'éviterons pas au cours de ce rapide et partiel survol de l'essai thérapeutique, inspiré d'une pratique cancérologique.

\section{Le difficile jeu de la vérité}

On ne parle plus de vérité au malade ; on doit plus modestement l'informer pour éclairer son consentement. Les mots ont été changés plus que le fond. Pudeur probable du médecin devant une impossible vérité. Le médecin doit s'interroger sur la capacité de son patient à comprendre les informations qu'il va lui communiquer. La complexité va croissant selon le type d'essai et l'état du sujet. Lorsqu'il s'agit d'un volontaire sain, la clarté, la précision des informations est d'une évidente nécessité, généralement acceptée par les acteurs. Qui prendrait le risque de ne pas mentionner les risques potentiels de l'essai ? L'absence de rémunération du volontaire et l'exaltation du geste altruiste dans la tradition française contraste avec le marché passé entre ceux qui proposent l'essai et ceux qui s'y prêtent contre rétribution, pratique anglo-saxonne admise et largement utilisée. Mais si le don de soi est aisément explicable lorsque l'enjeu médical est important, comment susciter les " bonnes volontés" lorsqu'il s'agit de traitements mineurs ou même de cosmétiques ? Dans l'essai thérapeutique classique où le patient espère un bénéfice direct du traitement auquel il se soumet, la communication de l'information s'impose, mais beaucoup plus difficilement qu'on ne pourrait l'imaginer. L'évaluation objective de la compétence du patient est un exercice technique [1]. Même si nous excluons le problème particulier des patients incompétents en raison de leur âge ou de leur état mental ou physique, il persiste plusieurs questions. Le médecin est-il capable de communiquer avec le patient? Le patient comprend-il l'information reçue? Est-il capable d'apprécier la situation et ses conséquences ? Est-il en état de prendre une véritable décision? On devine aisément comment et pourquoi la subjectivité s'introduit dans ce rapport humain. L'information ne sera sûrement pas communiquée de la même façon en France et aux États-Unis.

En France, à l'Institut GustaveRoussy au cours de mon internat dans les années 1960, nous n'osions pas prononcer le mot cancer devant les patients, car nous avions appris, en faculté de médecine, qu'il était contraire au code de déontologie de communiquer ce diagnostic au patient. Les temps ont changé et nous avec? Pourquoi ? Comment ? Probablement à cause de l'essai thérapeutique et de la naissance de l'espoir qu'il porte. Il est aussi frappant de voir comment on " assène " aisément le virus $\mathrm{HIV}$ et le SIDA par rapport à la pudique inhibition vis-à-vis du mot cancer qui persiste chez une partie des médecins. Nouveaux malades, nouveaux médecins probablement ; et surtout relations très différentes entre ces deux acteurs dans le cas du SIDA par rapport au cancer.

Mais la connaissance du diagnostic et du pronostic de la maladie ne suffit pas à effacer les problèmes éthiques que pose l'essai thérapeutique d'autant que la communauté médicale est souvent très divisée sur l'appréciation des effets des traitements. La multiplication depuis quelques $\mathrm{m} / \mathrm{s} n^{\circ}$ 5, vol. 7, mai 91 
années des conférences de consensus en cancérologie pourrait faire espérer une homogénéité dans la décision médicale face à une décision thérapeutique. L'étude de Moore, O'Sullivan et Tannock récemment publiée [2] démontre le poids de la subjectivité médicale influencée par la spécialité du médecin et le pays où il exerce. Ainsi six scénarios cliniques ont été proposés à des urologues canadiens, nord-américains et britanniques ainsi qu'à des radiothérapeutes et des chimiothérapeutes américains. Dans chaque scénario, le chirurgien ou le médecin devaient indiquer quel serait son choix thérapeutique s'il était lui-même atteint du cancer considéré (prostate, rein, vessie). Il est frappant de constater que le taux d'acceptation des essais cliniques randomisés par ces spécialistes a varié de 3 à $60 \%$, même dans des situations médicales apparemment bien codifiées. Par exemple, le radiothérapeute préfère nettement la radiothérapie du cancer de la prostate à la chirurgie, alors que les chirurgiens s'adonneraient à la prostatectomie. Aucun des deux ne fera appel à la chimiothérapie dans la plupart des cas. Les médecins britanniques sont beaucoup moins agressifs dans leurs choix thérapeutiques que leurs collègues nord-américains... Ainsi, les résultats de l'enquête décrite dans cet article, inspirée du " $\mathrm{Ne}$ fais pas à autrui ce que tu ne voudrais pas que l'on te fasse ", éclairent de manière troublante les choix thérapeutiques des médecins vis-à-vis de leurs patients.

Du coup, il faut se demander, ce qui n'est pas toujours clairement envisagé, si le médecin est capable d'effectuer cette évaluation technique de la compétence du patient. C'est d'abord un problème de formation médicale qui nous fait cruellement défaut. La faculté de médecine n'a pas alloué de temps à la préparation des médecins à l'essai thérapeutique. Certains peuvent arguer qu'une telle pratique est réservée au médecin hospitalier, formé sur le tas, dans un milieu où l'essai est monnaie courante ; mais il s'agit là d'une lacune dont le comblement serait bénéfique aussi bien pour le médecin hospitalier plein temps que pour le praticien de ville. Il est clair que ce dernier $\mathrm{m} / \mathrm{s} n^{\circ} 5$, vol. 7 , mai 91 joue un rôle certes indirect mais très important en faveur ou contre le succès de telle ou de telle nouvelle piste de recherche. Le développement de l'essai thérapeutique exige un effort pédagogique.

\section{Les enjeux du consentement}

La recherche a pour but de contribuer à la connaissance générale, ce qui exclut les pratiques non validées (en particulier, les médecines dites non orthodoxes ou parallèles). Le bénéfice du patient apparaît donc comme un sous-produit de l'essai thérapeutique justifiant ainsi le très classique aphorisme de Jean Bernard "L'essai thérapeutique est moralement nécessaire et nécessairement immoral ". Cette dimension de recherche introduit une tension entre le médecin et le malade, car le praticien devrait expliquer à ce dernier qu'il cherche d'abord la vérité scientifique qui couronnera ses efforts d'investigateur.

Voici le savoir devenu convoité et redouté. Il s'agit là d'une situation très délicate à appréhender correctement entre les principaux partenaires : le médecin, le patient et, autour, la famille et ses habitudes socioculturelles. De plus en plus clairement se dessinent en arrière-fond les enjeux du consentement.

Ceux-ci sont d'abord légaux. Il s'agit de préserver les droits du malade, mais, en même temps, de protéger le médecin des conséquences souvent imprévisibles, au moins pour les essais "lourds " faisant appel à des thérapeutiques agressives.

L'exploitation, à mon avis outrancière, aux États-Unis de la responsabilité médicale a dévié le consentement éclairé d'un acte de " communion " en une méthode de protection légale. C'est un contrat standardisé plus ou moins explicite qui est signé entre les partenaires. Cette évolution explique peut être aussi le rôle croissant des comités d'éthique soucieux de privilégier l'intérêt des patients par rapport aux autres acteurs [3]. A l'inverse, certains s'insurgent contre la nouvelle éthique qui prétend attribuer tous les droits au patient [4]. Ceux-là font valoir qu'en agissant ainsi on donne la priorité à qui choisit, et non pas au "bon choix "lui-même. A trop vouloir privilégier l'individu, objectent-ils, on favorise l'égoïsme par rapport à l'altruisme. Du coup, le médecin privé de parole a tendance à se désengager. Par exemple, les comités d'éthique souhaitent qu'en cas d'essai randomisé, le patient ait le droit de refuser le traitement auquel il a été assigné par tirage au sort et qu'il puisse exiger de recevoir le nouveau produit. On imagine aisément les problèmes posés par cette pratique au médecin, au statisticien, à la firme pharmaceutique soucieuse d'établir à coups de $p \leqslant 0,05$ la valeur de son produit [5]. Aussi faudra-t-il peut-être confronter l'éthique à la pratique pour en dégager progressivement le rôle et les limites dans la bonne conduite de l'essai thérapeutique [6, 7]. Mais si l'altruisme perd du terrain, il ne faut pas en accuser seulement les éthiciens et les patients. Chacun des partenaires n'attend-il pas son bénéfice? Le premier, pour le médecin, est la reconnaissance de sa valeur par ses confrères avec le prestige et éventuellement le pouvoir qui s'y rattachent. Vis-à-vis du malade et de sa famille, l'essai thérapeutique est bien entendu un moyen potentiel d'introduire l'espoir, élément essentiel de la relation médecin-malade. $\mathrm{Si}$ les avantages matériels que le médecin peut tirer de l'essai thérapeutique sont modestes, l'enjeu économique est grand pour les inventeurs et les producteurs des nouvelles thérapeutiques. L'industrie pharmaceutique est donc le partenaire obligatoire, de plus en plus contraignante parce qu'elle même contrainte, du médecin et du malade.

Les malades utiles à l'essai sont sélectionnés, car il faut avant tout se plier aux règles internationales d'autorisation de mise sur le marché de nouvelles drogues dans des indications strictement définies. Ainsi s'établit une chaîne d'interactions entre des intérêts économiques parfois contradictoires (entre les budgets de la santé et la diffusion de nouvelles molécules onéreuses) $[8,9]$ et des intérêts individuels.

Il est intéressant de rappeler le rôle majeur que les associations de patients ont joué pour l'accélération de la mise sur le marché de produits 
antiviraux destinés aux malades infectés par le virus HIV. Ils ont aussi perturbé l'essai thérapeutique en s'organisant pour détecter, par des moyens techniques mis en place par eux, le placebo du produit. Ces mouvements s'inscrivent dans le contexte plus général du glissement de l'offre de santé traditionnelle à la satisfaction des demandeurs et utilisateurs de la Santé [10]. Du coup, alors qu'en cancérologie les pouvoirs publics contrôlent bien l'autorisation de mise sur le marché, ils craignent de se voir dépassés sous la pression des malades dans le domaine du SIDA.

\section{La loi Huriet et la possible évolution des décisions de santé}

La loi dite Huriet, proposée par les sénateurs Huriet et Sérusclat, tente d'apporter des réponses aux problèmes que nous venons d'aborder. Elle propose la constitution d'équipes d'expérimentateurs spécialisés et reconnus, de façon à éliminer les mauvaises conduites d'essais par des expérimentateurs mal préparés à cette activité médicale.

La loi Huriet reste fidèle à la tradition française de l'absence de rémunération et rend obligatoire le consentement libre et éclairé, une pratique encore peu habituelle dans notre pays et qui devra être adaptée à notre contexte socioculturel de type latin.

Enfin la loi Huriet prend en compte les aspects socio-économiques de l'essai thérapeutique en exigeant la présentation d'un bilan coûtavantages du projet et en vérifiant simultanément sa validité scientifique et sociale. Les comités de protection des personnes devront veiller à son application.

Cette loi aura le mérite d'exister et de fournir à tous les intéressés un cadre légal précis. Mais elle risque aussi d'être rapidement confrontée à la probable évolution des rapports de force socioculturels capables d'influencer les décisions de santé. Ainsi, en créant des équipes d'expérimentateurs spécialisés, ne risquet-on pas de créer une nouvelle caste médicale toute-puissante et de se couper des médecins confinés au rôle de pourvoyeurs de patients? longtemps des comités de revue des protocoles et une surveillance des expérimentateurs, les cas de mauvaise conduite scientifique dans la pratique des essais thérapeutiques semblent relativement fréquents [11] : sur 964 audits de routine, plus de $10 \%$ montrent des déficiences dont $5,2 \%$ (50 investigateurs) sont considérées comme très sérieuses.

De plus, dans près de la moitié des études (61 \% des cas après 1988), le formalisme prévu du consentement éclairé n'avait pas été respecté; le protocole prévu n'avait pas été suivi dans plus de 100 cas et les données rapportées sur les formulaires n'étaient pas adéquates dans $22 \%$ des cas. Malgré ces insuffisances, la nécessité d'un contrôle des essais par des comités institutionnels est reconnue par les spécialistes de l'éthique des essais cliniques [12, 13].

Face à l'afflux attendu de nouvelles biothérapies grâce aux progrès de l'ingénierie génétique, les experts seront-ils en nombre suffisant pour permettre une autorisation de mise sur le marché rapide et médicalement justifiée?

Au souci des pouvoirs publics de contrôler l'augmentation des dépenses de santé s'opposera peut-être la hâte des usagers de disposer de nouvelles possibilités thérapeutiques, et des firmes pharmaceutiques de tirer profit des investissements de recherche importants que nécessite la production de nouvelles molécules actives. Dans le cas de maladies graves où l'arsenal disponible est très insuffisant, il faudra tenir compte de la pression considérable des patients et des familles. Elles soutiendront peut-être des médecins déçus de se sentir exclus de la possibilité de disposer des médicaments de l'espoir. Il suffit de noter les essais ouverts auxquels se sont prêtées des firmes pharmaceutiques dans le traitement du SIDA et qui ont abouti à confier à des médecins de famille, ou même directement aux patients, des produits n'ayant pas encore obtenu leur autorisation de mise sur le marché [14].

Du fait des difficultés croissantes d'enrôler des patients dans des essais randomisés, aux États-Unis, certaines firmes pharmaceutiques ont offert des rétributions à des médecins pour chaque malade adressé à l'investigateur et acceptant de rentrer dans l'essai [15].

Il apparaît donc que, dans ce pays où l'essai thérapeutique et ses aspects éthiques ont un passé solidement ancré sur une réglementation stricte, on assiste à une véritable " déstabilisation" de cette tradition sous le coup des évolutions récentes que nous venons de décrire. Devons-nous craindre que cette vague nous atteigne, avec quelque retard comme c'est souvent le cas pour les grands courants provenant d'outreAtlantique? Sommes-nous à l'abri de tels problèmes, aussi bien pour les patients que pour les médecins? Ce principe éthique auquel nous sommes attachés en France nous préservera-t-il de toute évolution néfaste ? Dans le meilleur des cas, il faut malgré tout s'attendre à de nombreuses difficultés dans l'application de la loi Huriet.

Les comités de protection des personnes seront probablement partagés entre leur souci de préserver à la fois les droits du patient et la qualité de l'évaluation médicale. Faudra-t-il envisager de nouvelles méthodes statistiques adaptées à des essais thérapeutiques ouverts où le grand nombre de patients traités peut apporter rapidement des informations précieuses sur le produit étudié ? Quel en sera le prix, dans tous les sens du mot ? Quoi qu'il en soit, la pédagogie médicale, et donc indirectement l'information des patients, devrait prendre en compte l'irruption probable de l'essai thérapeutique hors des filières d'experts.

$\mathrm{Au}$ fond, il ne sera plus question pour le malade de se contenter de donner un consentement éclairé pour se prêter à l'essai thérapeutique, mais d'exiger de disposer du produit puisqu'il est sujet et objet " éclairé " de l'essai. Le consentement est la réponse à une offre de soins par des médecins qui sera bientôt confrontée à une demande peut-être impérieuse des patients.

L'avenir nous éclairera bientôt sur l'évolution possible du consentement vers l'exigence des malades de prendre la responsabilité de leur choix thérapeutique, laissant aux médecins, aux statisticiens, aux juristes et aux spécialistes de l'éthique la tâche de s'adapter à cette demande 


\section{Summary}

The ethics of clinical trials : the dilemma of informed consent

The usual randomized clinical trials are now confronted by evolving legal, ethical and sociocultural factors, accelerated by the AIDS epidemic. In every case in which a new product is tested in a disease for which no therapy is clearly effective, the informed consent of the patient is required. This consent, however, does not conform to the wishes of the patient to choose his/her own treatment. It is becoming clear that physicians and statisticians must both take into consideration the wishes of the patient and safeguard the crucial distinction between research and current therapy. In France, the Hurict Law proposes for the first time, a legal framework for clinical trials, introducing the concept of specialized and authorised teams working under the supervision of local ethical committees. It is evident that cconomic considerations will influence the applications of this law.

TIRÉS A PART

C. Jasmin.

$m / s n^{\circ} 5$, vol. 7 , mai 91 